\title{
Towards the Next Generation of Recommender Systems
}

\author{
Alexander Tuzhilin \\ Professor of Information Systems and the NEC Faculty Fellow, Stern School of Business, \\ New York University
}

\begin{abstract}
The field of recommender systems has reached an important milestone in Fall 2009 when the $\$ 1,000,000$ Netflix Prize Competition has successfully completed its course. Since then, there has been an attempt made in the recommender systems community to access what has been accomplished and identify new promising research directions that the field can pursue after passing that milestone. In this talk, I will provide my personal perspective on what I think new important and promising research directions in the field are. I will also describe some of my projects pertaining to these directions, including context-aware, flexible and aggregation-based recommender systems.
\end{abstract}


